\title{
Asymptotic Tracking for Aircraft via Robust and Adaptive Dynamic Inversion Methods
}

\author{
W. MacKunis, P. M. Patre, M. K. Kaiser, and W. E. Dixon
}

\begin{abstract}
Two asymptotic tracking controllers are designed in this paper, which combine model reference adaptive control and dynamic inversion methodologies in conjunction with the robust integral of the signum of the error (RISE) technique for output tracking of an aircraft system in the presence of parametric uncertainty and unknown, nonlinear disturbances, which are not linearly parameterizable (non-LP). The control designs are complicated by the fact that the control input is multiplied by an uncertain, non-square matrix. A robust control design is presented first, in which partial knowledge of the aircraft model along with constant feedforward estimates of the unknown input parameters are used with a robust control term to stabilize the system. Motivated by the desire to reduce the need for high-gain feedback, an adaptive extension is then presented, in which feedforward adaptive estimates of the input uncertainty are used. These results show how asymptotic tracking control can be achieved for a nonlinear system in the presence of a non-square input matrix containing parametric uncertainty and nonlinear, non-LP disturbances. Asymptotic output tracking is proven via Lyapunov stability analysis, and high-fidelity simulation results are provided to verify the efficacy of the proposed controllers.
\end{abstract}

Index Terms-Adaptive control, aircraft tracking, dynamic Inversion, Lyapunov methods, nonlinear control, robust control.

\section{INTRODUCTION}

$\mathbf{F}$ EEDBACK linearization is a general control method where the nonlinear dynamics of a system are canceled by state feedback yielding a residual linear system. Dynamic inversion (DI) is a similar concept as feedback linearization that is commonly used within the aerospace community to replace linear aircraft dynamics with a reference model [1]-[8]. For example, in [3] a general DI approach is presented for a reference tracking problem for a minimum-phase and left-invertible linear system. A DI controller is designed for a nonminimum-phase hypersonic aircraft system in [1], which utilizes an additional controller to stabilize the zero dynamics. A finite-time stabilization design is proposed in [2], which utilizes DI given a full rank input matrix. Typically, DI methods assume the corresponding plant models are exactly known.

Manuscript received June 27, 2008. Manuscript received in final form December 21, 2009. First published February 02, 2010; current version published October 22, 2010. Recommended by Associate Editor J. Sarangapani. This work was supported in part by the NSF CAREER Award 0547448 and the Department of Energy Grant DE-FG04-86NE37967 as part of the DOE University Research Program in Robotics (URPR).

W. MacKunis and P. M. Patre are with the Department of Mechanical and Aerospace Engineering, University of Florida, Gainesville, FL 32611-6250 USA (e-mail: mackunis@gmail.com; pmpatre@ufl.edu).

M. K. Kaiser is with the Mosaic Atm, Inc., Leesburg, VA 20175 USA (e-mail: kkaiser@mosaicatm.com).

W. E. Dixon is with the Department of Mechanical and Aerospace Engineering, University of Florida, Gainesville, FL 32611-6250 USA (e-mail: wdixon@ufl.edu).

Digital Object Identifier 10.1109/TCST.2009.2039572
However, parametric uncertainty, additive disturbances, and unmodeled plant dynamics are always present in practical systems. Parametric uncertainty in the input matrix presents a particularly challenging scenario for DI controller design. Further complications arise in designing DI controllers for systems containing column-deficient input matrices.

For systems containing parametric uncertainty in the input matrix, inversion error is an inevitable challenge. Robust design methods are often utilized in DI controllers to compensate for parametric uncertainty and inversion error [9]-[12]. In [10], a stochastic robust DI technique is applied to a nonlinear aircraft model at high angle of attack. The controller in [10] is designed to compensate for uncertainties in the aerodynamic parameters, and is applicable to systems for which the nominal model is feedback linearizeable. In [11], a robust trajectory tracking controller is designed for an unmanned aerial vehicle (UAV) using a two-time-scaled dynamic inversion method. The controllers in [10] and [11] are based on the assumption that one subset of the state components evolves much faster than the other subset. While robust techniques can be utilized to stabilize systems containing parametric uncertainty, such techniques often require high gain or high frequency feedback to compensate for a worst case scenario of the uncertainty.

Motivated by the desire to adapt online for parametric uncertainty as opposed to designing based on the worst-case scenario, adaptive dynamic inversion (ADI) was also developed as a method to compensate for parametric uncertainty [3], [5], [6]. Typically, ADI methods exploit model reference adaptive control (MRAC) techniques, where the desired input-output behavior of the closed-loop system is given via the corresponding dynamics of a reference model [4], [6], [13]. Therefore, the basic task is to design a controller which will ensure the minimal error between the reference model and the plant outputs despite uncertainties in the plant parameters and working conditions.

Traditional Lyapunov-based adaptive control is applicable to systems with constant uncertainty that appears linearly in the system dynamics. However, several efforts (cf., [7], [14]-[17]) have been developed for the more general problem where the uncertain parameters or the inversion mismatch terms do not satisfy the linear-in-the-parameters assumption (i.e., non-LP). One method to compensate for non-LP uncertainty is to exploit a neural network as an online function approximation method as in [15] and [16]; however, all of these results yield uniformly ultimately bounded stability due to the inherent function reconstruction error. In [14] and [18], bounded aircraft tracking is proven using DI-based control strategies, where neural networks are utilized to compensate for model error. Motivated by the desire to achieve asymptotic tracking as opposed to bounded tracking, the control design presented here augments the typical RISE-based control structure with a feedforward element 
to compensate for parametric uncertainty in the input matrix. Further, the design presented here satisfies the need for a continuous controller, which is capable of achieving asymptotic output tracking for systems containing a column-deficient, uncertain input matrix and unmodeled nonlinear disturbances.

In contrast to neural network-based methods to compensate for the non-LP uncertainty, a robust control approach was recently developed in [19] (coined RISE control in [20]) to yield an asymptotic stability result. The RISE-based control structure has been used for a variety of fully actuated systems in [19], [21]-[28]. A contribution in this result is the use of the RISE control structure to achieve asymptotic output tracking control of a model reference system, where the plant dynamics include a bounded additive disturbance (e.g., potential disturbances include: gravity, inertial coupling, nonlinear gust modeling, etc.). This result represents application of the RISE method to a multiinput multi-output (MIMO) aircraft system containing a nonvanishing, non-LP disturbance, where the control input is multiplied by a column-deficient matrix containing parametric uncertainty. To achieve the result, the typical RISE control structure and closed-loop error system development are first modified by including a constant feedforward estimate of the input uncertainty along with a novel robust control term, which is designed to compensate for the resulting inversion error. Motivated by the desire to develop an adaptive controller, the robust control design is then extended to an ADI-based method, where a Lyapunov-based adaptive law is utilized to compensate for the parametric uncertainty in the input matrix. Asymptotic tracking results are proven via a Lyapunov stability analysis, and high fidelity numerical simulations are provided to show the performance of the proposed control designs. This current result is only applicable to relative degree 1 systems. Yet, results such as [21] and [29] provide inroads to apply the RISE control structure to systems of arbitrary relative degree.

The rest of the paper is organized as follows. In Section II, we introduce the aircraft model and present its mathematical properties. In Section III, a robust control design is presented, where a constant, best-guess feedforward estimate for the input uncertainty is used in the control law. A Lyapunov-based stability analysis of the robust control design is given in Section IV. In Section V, the control design is extended to an adaptive controller, where the motivation is to have an online adaptive feedforward estimate for the input uncertainty, as opposed to a constant estimate. In Section VI, the stability of the adaptive controller is proven via Lyapunov-based analysis, and numerical simulation results are presented in Section VII to complement the theoretical development.

\section{Aircraft Model AND PROPERTIES}

The aircraft system under consideration in this paper can be modeled via the following state space representation [1], [5], [8], [30], [31]:

$$
\dot{x}=A x+B u+f(x, t) \quad y=C x
$$

where $A \in \mathbb{R}^{n \times n}$ denotes the uncertain state matrix, $B \in \mathbb{R}^{n \times m}$ for $m<n$ represents the uncertain input matrix, $C \in \mathbb{R}^{m \times n}$ is the known output matrix, $u \in \mathbb{R}^{m}$ is a vector of control inputs, ${ }^{1}$ and $f(x, t) \in \mathbb{R}^{n}$ represents an unknown, nonlinear disturbance.

Assumption 1: The nonlinear disturbance $f(x, t)$ and its first two time derivatives are assumed to exist and be bounded by known constants.

Assumption 2: A large magnitude disturbance (e.g., wind gust) could cause the aircraft to become unstable or uncontrollable; however, the subsequent development is based on the assumption that the dynamics in (1) are controllable.

The disturbance $f(x, t)$ introduced in (1) can represent several bounded nonlinearities. For smaller aircraft flying around trim, an example of a bounded nonlinear disturbance that can be represented by $f(x, t)$ in (1) is a sudden, individual gust. The model given in [32], for example, defines such a bounded nonlinearity along the longitudinal axis (see [9] for details). A similar model can also be used to define bounded nonlinearities due to lateral gusts.

\section{Robust CONTROL DEVElopMENT}

A contribution of the development in this section is a robust technique that yields asymptotic tracking for an aircraft in the presence of parametric uncertainty in a non-square input matrix and an unknown nonlinear disturbance. To this end, the control law is developed based on the output dynamics, which enables us to transform the uncertain input matrix into a square matrix. By utilizing a feedforward (best guess) estimate of the input uncertainty in the control law in conjunction with a robust control term, we are able to compensate for the input uncertainty. Specifically, asymptotic tracking is proven based on the assumption that an estimate of the uncertain input matrix can be selected such that a diagonal dominance property is satisfied in the closed-loop error system.

\section{A. Error System}

The control objective in this section is to ensure that the system outputs track desired time-varying reference outputs despite unknown, nonlinear, non-LP disturbances in the dynamic model. To quantify this objective, a tracking error, denoted by $e(t) \in \mathbb{R}^{m}$, is defined as

$$
e \triangleq y-y_{m}=C\left(x-x_{m}\right)
$$

where the reference state $x_{m}(t) \in \mathbb{R}^{n}$ and reference output $y_{m}(t) \in \mathbb{R}^{m}$ are generated by the following reference model:

$$
\dot{x}_{m}=A_{m} x_{m}+B_{m} \delta \quad y_{m}=C x_{m}
$$

where the reference state and input matrices are denoted by $A_{m} \in \mathbb{R}^{n \times n}$ and $B_{m} \in \mathbb{R}^{n \times m}$, respectively, and $\delta(t) \in \mathbb{R}^{m}$ denotes an input to the reference model. To facilitate the subsequent analysis, a filtered tracking error [33], denoted by $r(t) \in$ $\mathbb{R}^{m}$, is defined as

$$
r \triangleq \dot{e}+\alpha e
$$

\footnotetext{
${ }^{1}$ The subsequent development assumes that $u(t) \in \mathbb{R}^{m}$ and $C \in \mathbb{R}^{m \times n}$ (i.e., the number of inputs is equal to the number of outputs). However, this control design can be applied to systems for which $u(t) \in \mathbb{R}^{p}$ and $C \in \mathbb{R}^{m \times n}$, where $p>m$ via the use of a pseudoinverse (e.g., Moore-Penrose) in the control law.
} 
where $\alpha \in \mathbb{R}^{m \times m}$ denotes a positive definite, diagonal matrix of constant control gains. The subsequent development is based on the assumption that $y(t)$ and $\dot{y}(t)$ are measurable, and hence, $e(t), \dot{e}(t)$, and $r(t)$ are measurable. While the requirement for these measurements is a potential deficit, sensors can be used to provide this feedback (e.g., see [34]-[38] for aircraft controllers with similar feedback requirements). The time derivative $\dot{y}(t)$ contains $\dot{q}(t), \dot{p}(t), \dot{r}(t)$, and the acceleration along the flight path, where $q(t), p(t)$, and $r(t)$ denote the pitch, roll, and yaw rates, respectively. Angular accelerometers could realistically be used to measure $\dot{q}(t), \dot{p}(t)$, and $\dot{r}(t)$ online, but measuring the true value of the acceleration along the flight path would be difficult in practice since it depends on the aircraft velocity $w(t)$ in the body $z$-direction. It is assumed in this paper that the time derivative $\dot{y}(t)$ can be estimated by combining an estimate of $w(t)$ (e.g., using an angle of attack estimator and an air data system such as a pitot static tube) with an axial accelerometer measurement.

Assumption 3: The unmeasurable states $x_{u}(t) \in \mathbb{R}^{n-m}$ in (1) and the corresponding time derivatives are bounded in terms of the measurable error signal such that the following inequalities hold:

$$
\left\|x_{u}\right\| \leq c_{1}\|z\|+\zeta_{x u} \quad\left\|\dot{x}_{u}\right\| \leq c_{2}\|z\|+\zeta_{\dot{x} u}
$$

where $z(t) \in \mathbb{R}^{2 m}$ is defined as

$$
z \triangleq\left[\begin{array}{ll}
e^{T} & r^{T}
\end{array}\right]^{T}
$$

and $c_{1}, c_{2}, \zeta_{x u}, \zeta_{\dot{x} u} \in \mathbb{R}$ are known nonnegative bounding constants (i.e., the constants could be set to zero). This assumption is tantamount to assuming that the system in (1) is detectable.

The open-loop tracking error dynamics can be developed by taking the time derivative of (4) and utilizing the expressions in (1) and (3) to obtain the following expression:

$$
\dot{r}=\tilde{N}+N_{d}+\Omega(\dot{u}+\alpha u)-e
$$

where the auxiliary function $\tilde{N}(x, \dot{x}, e, \dot{e}) \in \mathbb{R}^{m}$ is defined as

$$
\tilde{N} \triangleq C A\left(\dot{x}_{o}-\dot{x}_{o m}\right)+\alpha C A\left(x_{o}-x_{o m}\right)+C A\left(\dot{x}_{\rho u}+\alpha x_{\rho u}\right)+e
$$

the auxiliary function $N_{d}\left(x_{m}, \dot{x}_{m}, \delta, \dot{\delta}\right) \in \mathbb{R}^{m}$ is defined as

$$
\begin{aligned}
N_{d} \triangleq & C A\left(\dot{x}_{o m}+\alpha x_{o m}\right)+C(\dot{f}(x, t)+\alpha f(x, t)) \\
& -C A_{m}\left(\dot{x}_{m}+\alpha x_{m}\right)-C B_{m}(\dot{\delta}+\alpha \delta) \\
& +C A\left(\dot{x}_{\zeta u}+\alpha x_{\zeta u}\right)
\end{aligned}
$$

and the constant, unknown matrix $\Omega \in \mathbb{R}^{m \times m}$ is defined as

$$
\Omega \triangleq C B
$$

In (8) and (9), $x_{\rho u}(t), \dot{x}_{\rho u}(t) \in \mathbb{R}^{n}$ contain the portions of $x_{u}(t)$ and $\dot{x}_{u}(t)$, respectively, that can be upper bounded by functions of $z(t) ; x_{\zeta u}(t), \dot{x}_{\zeta u}(t) \in \mathbb{R}^{n}$ contain the portions of $x_{u}(t)$ and $\dot{x}_{u}(t)$ that can be upper bounded by known constants [i.e., see (5)]; $x_{o}(t) \in \mathbb{R}^{n}$ contains the measurable states (i.e., $\left.x(t)=x_{o}(t)+x_{\rho u}(t)+x_{\zeta u}(t)\right)$; and $x_{o m}(t) \in \mathbb{R}^{n}$ contains the reference states corresponding to the measurable states $x_{o}(t)$. The quantities $\tilde{N}(x, \dot{x}, e, \dot{e})$ and $N_{d}\left(x_{m}, \dot{x}_{m}, \delta, \dot{\delta}\right)$ and the derivative $\dot{N}_{d}\left(x_{m}, \dot{x}_{m}, \ddot{x}_{m}, \delta, \dot{\delta}, \ddot{\delta}\right)$ can be upper bounded as follows [19], [24]:

$$
\|\tilde{N}\| \leq \rho(\|z\|)\|z\|, \quad\left\|N_{d}\right\| \leq \zeta_{N_{d}}, \quad\left\|\dot{N}_{d}\right\| \leq \zeta_{\dot{N}_{d}}
$$

where $\zeta_{N_{d}}, \zeta_{\dot{N}_{d}} \in \mathbb{R}$ are known positive bounding constants, and the function $\rho(\|z\|)$ is a positive, globally invertible, nondecreasing function. Based on (10), a constant feedforward estimate $\hat{\Omega} \in \mathbb{R}^{m \times m}$ is defined as

$$
\hat{\Omega}=C \hat{B}
$$

where $\hat{B}$ is a constant, best-guess estimate of the uncertain matrix $B$. The constant, user-defined matrix $\hat{\Omega}$ is assumed to be invertible. Based on the expression in (7) and the subsequent stability analysis, the control input is designed as

$$
\begin{aligned}
u= & -\alpha \int_{0}^{t} u(\tau) d \tau-\int_{0}^{t} \hat{\Omega}^{-1} k_{\gamma} \operatorname{sgn}(r(\tau)) d \tau \\
& -\hat{\Omega}^{-1} \int_{0}^{t}\left[\left(k_{s}+I_{m \times m}\right) r(\tau)+\beta \operatorname{sgn}(e(\tau))\right] d \tau
\end{aligned}
$$

where $\beta, k_{s}, k_{\gamma} \in \mathbb{R}^{m \times m}$ are positive definite, diagonal matrices of constant control gains, $\alpha$ was defined in (4), and $I_{m \times m} \in \mathbb{R}^{m \times m}$ denotes the $m \times m$ identity matrix. To simplify the notation in the subsequent stability analysis, the constant auxiliary matrix $\tilde{\Omega} \in \mathbb{R}^{m \times m}$ is defined as

$$
\tilde{\Omega} \triangleq \Omega \hat{\Omega}^{-1}
$$

where $\tilde{\Omega}$ can be separated into diagonal (i.e., $\Lambda \in \mathbb{R}^{m \times m}$ ) and off-diagonal (i.e., $\Delta \in \mathbb{R}^{m \times m}$ ) components as

$$
\tilde{\Omega}=\Lambda+\Delta \text {. }
$$

After substituting the time derivative of (13) into (7), the following closed-loop error system is obtained:

$\dot{r}=\tilde{N}+N_{d}-\tilde{\Omega}\left(k_{s}+I_{m \times m}\right) r-\tilde{\Omega} k_{\gamma} \operatorname{sgn}(r)-\tilde{\Omega} \beta \operatorname{sgn}(e(t))-e$.

Assumption 4: The subsequent development is based on the assumption that the constant estimate $\hat{\Omega}$ given in (12) can be selected as

$$
\lambda_{\min }(\Lambda)-\|\Delta\|_{i \infty}>\varepsilon,
$$

where $\varepsilon \in \mathbb{R}$ is a known positive constant, $\|\cdot\|_{i \infty}$ denotes the induced infinity norm of a matrix, and $\lambda_{\min }(\cdot)$ denotes the minimum eigenvalue of the argument. Preliminary testing results show this assumption is mild in the sense that (17) is satisfied for a wide range of $\hat{\Omega} \neq \Omega$.

\section{Stability Analysis}

Let $\mathcal{D} \subset \mathbb{R}^{2 m+1}$ be a domain containing $w(t)=0$, where $w(t) \in \mathbb{R}^{2 m+1}$ is defined as

$$
w(t) \triangleq\left[\begin{array}{ll}
z^{T} & \sqrt{P(t)}
\end{array}\right]^{T}
$$

and the auxiliary function $P(t) \in \mathbb{R}$ is defined as

$$
\begin{aligned}
P(t) \triangleq & \|\beta\|_{i \infty}\|e(0)\|\|\Lambda\|_{i \infty}-e(0)^{T} N_{d}(0) \\
& +\sqrt{m} \int_{0}^{t}\|\beta\|_{i \infty}\|\Delta\|_{i \infty}\|r(\tau)\| d \tau-\int_{0}^{t} L(\tau) d \tau
\end{aligned}
$$


The auxiliary function $L(t) \in \mathbb{R}$ in (19) is defined as

$$
L(t) \triangleq r^{T}\left(N_{d}(t)-\tilde{\Omega} \beta \operatorname{sgn}(e)\right) .
$$

The following lemma is provided to facilitate the main result in Theorem 1.

Lemma 1: Provided the sufficient gain condition

$$
\|\beta\|_{i \infty}>\frac{\left(\zeta_{N_{d}}+\frac{1}{\|\alpha\|_{i \infty}} \zeta_{\dot{N}_{d}}\right)}{\lambda_{\min }(\Lambda)}
$$

is satisfied, the following inequality can be obtained:

$$
\begin{aligned}
\int_{0}^{t} L(\tau) d \tau \leq & \|\beta\|_{i \infty}\|e(0)\|\|\Lambda\|_{i \infty}-e(0)^{T} N_{d}(0) \\
& +\sqrt{m} \int_{0}^{t}\|\beta\|_{i \infty}\|\Delta\|_{i \infty}\|r(\tau)\| d \tau .
\end{aligned}
$$

Hence, (22) can be used to conclude that $P(t) \geq 0$.

Theorem 1: The controller given in (13) ensures that the output tracking error is regulated in the sense that

$$
\|e(t)\| \rightarrow 0 \text { as } t \rightarrow \infty
$$

provided the control gain $k_{s}$ introduced in (13) is selected sufficiently large (see the subsequent stability proof), $\beta$ is selected to satisfy (21), and $k_{\gamma}$ is selected according to the following sufficient condition:

$$
\lambda_{\min }\left(k_{\gamma}\right)>\frac{\sqrt{m}\|\beta\|_{i \infty}\|\Delta\|_{i \infty}}{\varepsilon}
$$

where $\zeta_{N_{d}}$ and $\zeta_{\dot{N}_{d}}$ are introduced in (11), $\varepsilon$ is defined in (17), and $\Lambda$ and $\Delta$ are introduced in (15).

Proof: Let $V(w, t): \mathcal{D} \times[0, \infty) \rightarrow \mathbb{R}$ be a continuously differentiable, positive definite function defined as

$$
V \triangleq \frac{1}{2} e^{T} e+\frac{1}{2} r^{T} r+P
$$

where $e(t)$ and $r(t)$ are defined in (2) and (4), respectively, and the positive definite (see Lemma 1) function $P(t)$ is defined in (19). The positive definite function $V(w, t)$ satisfies the inequality

$$
U_{1}(w) \leq V(w, t) \leq U_{2}(w)
$$

provided the sufficient condition introduced in (21) is satisfied. In (26), the continuous, positive definite functions $U_{1}(w), U_{2}(w) \in \mathbb{R}$ are defined as

$$
U_{1} \triangleq \eta_{1}\|w\|^{2} \quad U_{2} \triangleq \eta_{2}\|w\|^{2}
$$

where $\eta_{1}=(1 / 2)$, and $\eta_{2}=1$. After taking the time derivative of (25) and utilizing (4), (15), (16), (19), and (20), $\dot{V}(w, t)$ can be expressed as

$$
\begin{aligned}
\dot{V}(w, t)= & -e^{T} \alpha e+r^{T} \tilde{N}+\sqrt{m}\|\beta\|_{i \infty}\|r\|\|\Delta\|_{i \infty} \\
& -r^{T} \Delta\left(k_{s}+I_{m \times m}\right) r-r^{T} \Lambda\left(k_{s}+I_{m \times m}\right) r \\
& -r^{T} \Delta k_{\gamma} \operatorname{sgn}(r)-r^{T} \Lambda k_{\gamma} \operatorname{sgn}(r) .
\end{aligned}
$$

By utilizing (11), $\dot{V}(w, t)$ can be upper bounded as

$$
\begin{aligned}
\dot{V}(w, t) \leq & -\lambda_{\min }(\alpha)\|e\|^{2}-\varepsilon\|r\|^{2}-\lambda_{\min }\left(k_{s}\right) \varepsilon\|r\|^{2} \\
& +\left[-\lambda_{\min }\left(k_{\gamma}\right) \varepsilon+\sqrt{m}\|\beta\|_{i \infty}\|\Delta\|_{i \infty}\right]\|r\| \\
& +\rho(\|z\|)\|r\|\|z\| .
\end{aligned}
$$

If the sufficient gain condition in (24) is satisfied, the bracketed term in (29) is negative, and $\dot{V}(w, t)$ can be upper bounded using the squares of the components of $z(t)$ as follows:

$$
\begin{aligned}
\dot{V}(w, t) \leq & -\lambda_{\min }(\alpha)\|e\|^{2}-\varepsilon\|r\|^{2}+[\rho(\|z\|)\|r\|\|\mid\| z \| \\
& \left.-\lambda_{\min }\left(k_{s}\right) \varepsilon\|r\|^{2}\right] .
\end{aligned}
$$

Completing the squares for the bracketed terms in (30) yields

$$
\dot{V}(w, t) \leq-\eta_{3}\|z\|^{2}+\frac{\rho^{2}(\|z\|)\|z\|^{2}}{4 \lambda_{\min }\left(k_{s}\right) \varepsilon}
$$

where $\eta_{3} \triangleq \min \left\{\lambda_{\min }(\alpha), \varepsilon\right\}$, and $\rho(\|z\|)$ is introduced in (11). The following expression can be obtained from (31):

$$
\dot{V}(w, t) \leq-U(w)
$$

where $U(w)=c\|z\|^{2}$, for some positive constant $c \in \mathbb{R}$, is a continuous, positive semi-definite function that is defined on the following domain:

$$
\mathcal{D} \triangleq\left\{w \in \mathbb{R}^{2 m+1} \mid\|w\|<\rho^{-1}\left(2 \sqrt{\eta_{3} \lambda_{\min }\left(k_{s}\right) \varepsilon}\right)\right\} .
$$

The inequalities in (26) and (32) can be used to show that $V(t) \in$ $\mathcal{L}_{\infty}$ in $\mathcal{D}$; hence $e(t), r(t) \in \mathcal{L}_{\infty}$ in $\mathcal{D}$. Given that $e(t), r(t) \in$ $\mathcal{L}_{\infty}$ in $\mathcal{D}$, standard linear analysis methods can be used to prove that $\dot{e}(t) \in \mathcal{L}_{\infty}$ in $\mathcal{D}$ from (4). Since $e(t), \dot{e}(t) \in \mathcal{L}_{\infty}$ in $\mathcal{D}$, the assumption that $y_{m}, \dot{y}_{m} \in \mathcal{L}_{\infty}$ in $\mathcal{D}$ can be used along with (2) to prove that $y, \dot{y} \in \mathcal{L}_{\infty}$ in $\mathcal{D}$. Given that $r(t) \in \mathcal{L}_{\infty}$ in $\mathcal{D}$, the assumption that $\hat{\Omega}^{-1} \in \mathcal{L}_{\infty}$ in $\mathcal{D}$ can be used along with the time derivative of (13) to show that $\dot{u}(t) \in \mathcal{L}_{\infty}$ in $\mathcal{D}$. Further, Equation 2.78 of [39] can be used along with the fact that $\alpha$ is diagonal to show that the elements $\dot{u}_{i}(t) \in \mathbb{R}, \forall i=1, \ldots, m$, of $\dot{u}(t)$ can be upper bounded as $\dot{u}_{i}(t) \leq-\alpha_{i} u_{i}(t)+M_{i}, \forall t \geq$ 0 , where $M_{i} \in \mathbb{R}^{+}, \forall i=1, \ldots, m$, are bounding constants, and $\alpha_{i}, \forall i=1, \ldots, m$, denotes the $i$ th diagonal element of $\alpha$. Reference [40, Th. 1.1] can then be utilized to show that $u(t) \in$ $\mathcal{L}_{\infty}$ in $\mathcal{D}$. Hence, (16) can be used to show that $\dot{r}(t) \in \mathcal{L}_{\infty}$ in $\mathcal{D}$. Since $\dot{e}(t), \dot{r}(t) \in \mathcal{L}_{\infty}$ in $\mathcal{D}$, the definitions for $U(w)$ and $z(t)$ can be used to prove that $U(w)$ is uniformly continuous in $\mathcal{D}$.

Let $S \subset \mathcal{D}$ denote a set defined as follows:

$$
S \triangleq\left\{w(t) \subset \mathcal{D} \mid \eta_{2}\|w\|^{2}<\eta_{1}\left(\rho^{-1}\left(2 \sqrt{\eta_{3} \lambda_{\min }\left(k_{s}\right) \varepsilon}\right)\right)^{2}\right\} .
$$

Reference [41, Th. 8.4] can now be invoked to state that

$$
c\|z\|^{2} \rightarrow 0 \quad \text { as } \quad t \rightarrow \infty \quad \forall w(0) \in S .
$$

Based on the definition of $z$, (35) can be used to show that

$$
\|e(t)\| \rightarrow 0 \quad \text { as } \quad t \rightarrow \infty \quad \forall w(0) \in S .
$$




\section{AdAPTIVE CONTROL EXTENSION}

The robust DI control technique presented in the previous sections can be extended to an ADI-based control method. Robust control methods can be utilized to compensate for both structured and unstructured bounded uncertainty; however, since robust control methods are based on worst-case uncertainty and disturbances, high gain or high frequency feedback is often required to achieve stability. The subsequent analysis illustrates how asymptotic output tracking can be achieved for the aircraft system in (1) by utilizing a Lyapunov-based adaptive law to compensate for the parametric uncertainty in the input matrix.

Assumption 5: The matrix product $C B$ is assumed to be invertible. The invertibility condition means that the control development and stability analysis presented in this paper is applicable to relative degree 1 systems.

\section{A. Error System}

After taking the time derivative of (4) and utilizing the expressions in (1) and (3), the open-loop error dynamics can be expressed as

$$
\dot{r}=\tilde{N}+N_{d}+Y_{B} \tilde{\theta}_{B}+C \hat{B}(\dot{u}+\alpha u)-e .
$$

In (37), the auxiliary functions $\tilde{N}\left(x, \dot{x}, e, x_{m}, \dot{x}_{m}, t\right) \in \mathbb{R}^{m}$ and $N_{d}\left(x_{m}, \dot{x}_{m}, \delta, \dot{\delta}, t\right) \in \mathbb{R}^{m}$ are defined as

$$
\begin{aligned}
\tilde{N} \triangleq & C\left(\dot{f}(x, \dot{x}, t)-\dot{f}\left(x_{m}, \dot{x}_{m}, t\right)\right) \\
& +C A\left((\dot{x}+\alpha x)-\left(\dot{x}_{m}+\alpha x_{m}\right)\right)+e \\
& +\alpha C f(x, t)-\alpha C f\left(x_{m}, t\right) \\
N_{d} \triangleq & -C\left(A_{m}-A\right)\left(\dot{x}_{m}+\alpha x_{m}\right)-C B_{m}(\dot{\delta}+\alpha \delta) \\
& +C\left(\dot{f}\left(x_{m}, \dot{x}_{m}, t\right)+\alpha f\left(x_{m}, t\right)\right) .
\end{aligned}
$$

Motivation for the selective grouping of the terms in $\tilde{N}$ and $N_{d}$ is derived from the fact that (38) and (39) can be shown to satisfy (11). Also in (37), $Y_{B}(u, \dot{u}) \in \mathbb{R}^{m \times p_{1}}$ denotes a measurable regression matrix, and $\theta_{B} \in \mathbb{R}^{p_{1}}$ is a vector containing the unknown parameters of the $B$ matrix, defined via the parameterization

$$
Y_{B} \theta_{B} \triangleq C B(\dot{u}+\alpha u) .
$$

In (37) and the subsequent stability analysis, $\tilde{\theta}_{B}(t) \in \mathbb{R}^{p_{1}}$ denotes the parameter estimation error defined as

$$
\tilde{\theta}_{B} \triangleq \theta_{B}-\hat{\theta}_{B}
$$

where $\hat{\theta}_{B}(t) \in \mathbb{R}^{p_{1}}$ denotes a subsequently designed parameter estimate vector. The estimate matrix $\hat{B}(t) \in \mathbb{R}^{n \times m}$ is introduced in (37) to facilitate the control development, where the elements of the matrix are composed of the elements of $\hat{\theta}_{B}(t)$.

Based on the expression in (37) and the subsequent stability analysis, the control input is designed as

$$
u=-\int_{0}^{t}[\alpha u(\tau)+\Omega(\tau)] d \tau
$$

where the auxiliary function $\Omega(t) \in \mathbb{R}^{m}$ is defined as

$$
\Omega \triangleq(C \hat{B})^{-1}\left[\left(k_{s}+I_{m \times m}\right) r+\beta \operatorname{sgn}(e)\right]
$$

where $\beta, k_{s} \in \mathbb{R}^{m \times m}$ are defined in (13), and $\alpha$ is defined in (4). The adaptive estimate $\hat{B}(t)$ (or $\hat{\theta}_{B}(t)$ in vector form) in (43) is generated online according to the adaptive update law

$$
\dot{\hat{\theta}}_{B}=\operatorname{proj}\left\{\Gamma_{B} Y_{B}^{T} r\right\} .
$$

In (44), $\Gamma_{B} \in \mathbb{R}^{p_{1} \times p_{1}}$ is a constant, positive definite, symmetric adaptation gain matrix, and $\operatorname{proj}(\cdot)$ denotes a projection operator utilized to guarantee that the $i$ th element of $\hat{\theta}_{B}(t)$ is bounded as

$$
\underline{\theta}_{B i} \leq \hat{\theta}_{B i} \leq \bar{\theta}_{B i}
$$

where $\bar{\theta}_{B i}, \underline{\theta}_{B i} \in \mathbb{R}$ denote known, constant upper and lower bounds, respectively, for each element of $\hat{\theta}_{B}(t)$. On an element by element basis, the projection operator in (44) can be used to ensure that the matrix estimate $C \hat{B}(t)$ is invertible under the assumption that $C B$ is invertible [1], [8], [42] for all elements of $B$ contained within some bounded region (i.e., $\underline{\theta}_{B i} \leq \theta_{B i} \leq$ $\bar{\theta}_{B i}$ ). After substituting the time derivative of (42) into (37), the closed-loop error system can be determined as

$$
\dot{r}=\tilde{N}+N_{d}+Y_{B} \tilde{\theta}_{B}-\left(k_{s}+I_{m \times m}\right) r-\beta s g n(e)-e .
$$

\section{STABILITY ANALYSIS}

Let $\mathcal{D} \subset \mathbb{R}^{2 m+p_{1}+1}$ be a domain containing $w(t)=0$, where $w(t) \in \mathbb{R}^{2 m+p_{1}+1}$ is defined as

$$
w(t) \triangleq\left[\begin{array}{lll}
z^{T}(t) & \tilde{\theta}_{B}^{T}(t) & \sqrt{P(t)}
\end{array}\right]^{T}
$$

where the auxiliary function $P(t) \in \mathbb{R}$ is defined as

$$
P(t) \triangleq\|\beta\|_{i \infty}\|e(0)\|-e(0)^{T} N_{d}(0)-\int_{0}^{t} L(\tau) d \tau
$$

where the auxiliary function $L(t) \in \mathbb{R}$ is defined as

$$
L(t) \triangleq r^{T}\left(N_{d}(t)-\beta \operatorname{sgn}(e)\right) .
$$

The following lemma is provided to facilitate the main result in Theorem 2 .

Lemma 2: Provided $\beta$ is selected according to the sufficient condition

$$
\|\beta\|_{i \infty}>\zeta_{N_{d}}+\frac{1}{\|\alpha\|_{i \infty}} \zeta_{\dot{N}_{d}}
$$

where $\zeta_{N_{d}}$ and $\zeta_{\dot{N}_{d}}$ were introduced in (11), the following inequality can be obtained:

$$
\int_{0}^{t} L(\tau) d \tau \leq\|\beta\|_{i \infty}\|e(0)\|-e(0)^{T} N_{d}(0)
$$

Hence, (51) can be used to conclude that $P(t) \geq 0$.

Proof: See [19], [20] for proof of Lemma 2. 

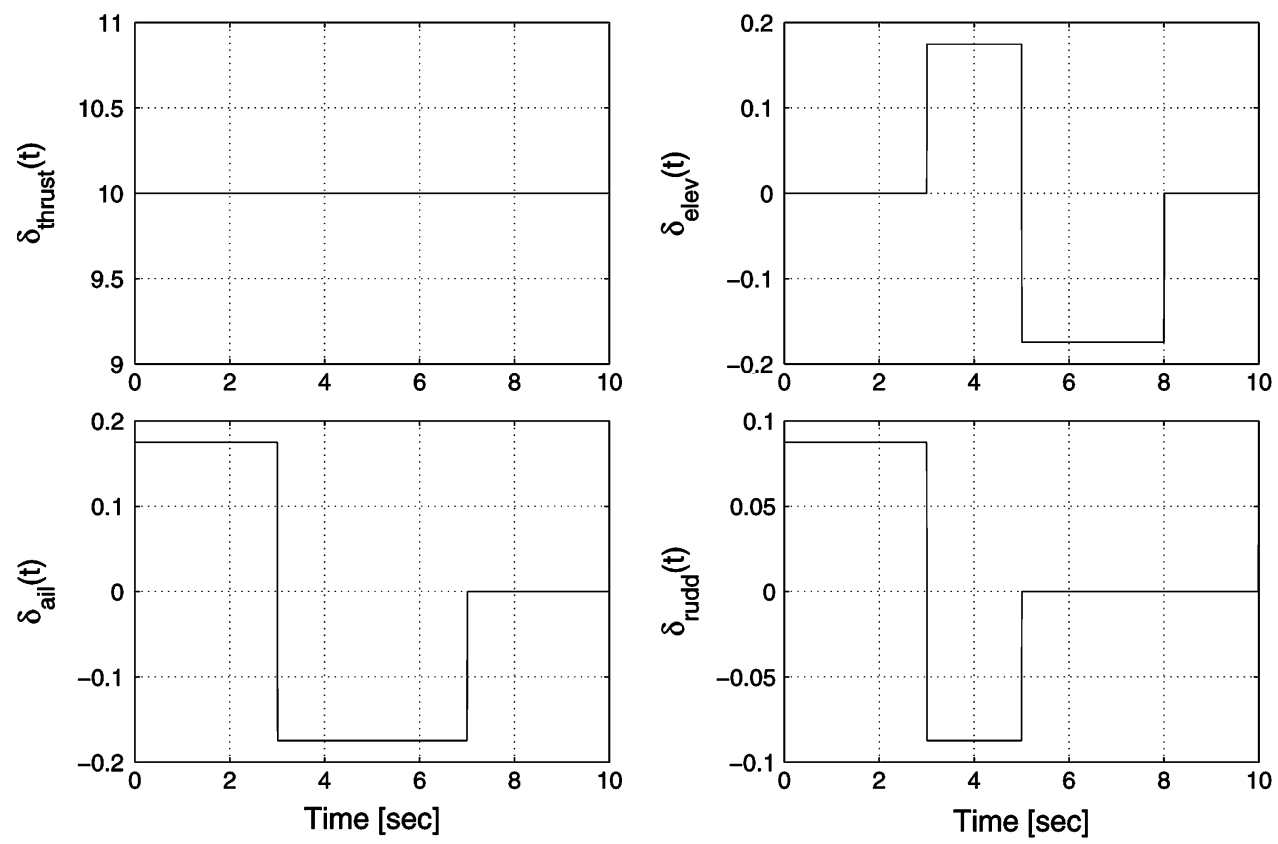

Fig. 1. Reference inputs away from trim. Thrust (top left), elevator (top right), aileron (bottom left), and rudder (bottom right) reference commands used for the robust and adaptive controller simulations.

Theorem 2: The controller given in (42) ensures that the output tracking error is regulated in the sense that $\|e(t)\| \rightarrow 0$ as $t \rightarrow \infty$, provided the control gain matrix $k_{s}$ introduced in (43) is selected sufficiently large, and the sufficient condition in (50) is satisfied.

Proof: The proof of Theorem 2 is similar to that given in Section IV and [43] and is omitted here for brevity.

\section{SimULATION RESULTS}

\section{A. Simulation Model}

Numerical simulations were created to test the efficacy of the developed controllers. While numerical simulation results are being presented as opposed to experimental results, the sensor noise, actuator saturation and rate limits, and disturbances used in the numerical simulation are based on detailed analyses and specifications from typical sensors and actuators for an Osprey UAV. Table I of [9], [43] shows the simulation parameters used that correspond to these hardware constraints. The simulations are based on the Osprey fixed wing aerial vehicle, for which the model can be represented using the state space system given in (1), where $n=8$, and $m=4$. The lateral mode is represented using four states. The yaw angle is left out because it imparts no accelerations to the aircraft [44]. The numerical values used for the state and input matrices $A \in \mathbb{R}^{8 \times 8}$ and $B \in \mathbb{R}^{8 \times 4}$ are based on experimentally determined data for the Osprey aircraft at a cruising velocity of $25 \mathrm{~m} / \mathrm{s}$ at an altitude of $60 \mathrm{~m}$. Based on the standard assumption that the longitudinal and lateral modes of the aircraft can be decoupled, the state matrix $A \in \mathbb{R}^{8 \times 8}$ and input matrix $B \in \mathbb{R}^{8 \times 4}$ can be expressed in block diagonal form, and the input and output vectors can be segregated in terms of the longitudinal and lateral subsystems (for details on the structure of $A, B, x, y$, and $u$, see [9] and [43]). The reference model for the simulation is represented by the reference state space system given in (3), where the state matrix $A_{m}$ and input matrix $B_{m}$ are designed with the specific purpose of decoupling the forward velocity and pitch rate within the longitudinal system as well as decoupling the roll rate and yaw rate within the lateral system.

In (1), the nonlinear disturbance term $f(x, t) \triangleq$ $\left[f\left(x_{\text {lon }}, t\right)^{T}, f\left(x_{\text {lat }}, t\right)^{T}\right]^{T}$, where $f\left(x_{\text {lon }}, t\right), f\left(x_{\text {lat }}, t\right) \in \mathbb{R}^{4}$ are defined as

$$
\begin{aligned}
f\left(x_{\text {lon }}, t\right) & =\left[\begin{array}{llll}
-9.81 \sin \theta & 0 & 0 & 0
\end{array}\right]^{T}+g(x)^{T} \\
f\left(x_{\text {lat }}, t\right) & =\left[\begin{array}{llll}
0.39 \sin \phi & 0 & 0 & 0
\end{array}\right]^{T}
\end{aligned}
$$

where $g(x) \in \mathbb{R}^{4}$ represents a disturbance due to a sudden, individual gust as defined in [32]. The remainder of the additive disturbances in (52) and (53) represent nonlinearities not captured in the linearized state space model (e.g., due to small angle assumptions). All states and control inputs were initialized to zero for the simulation. To test the scenario in which the input parameters are uncertain, the adaptive estimates were initialized to values that differ from the true parameter values by approximately $60 \%$. For the robust DI controller presented in Section III, the constant feedforward estimates $\hat{B}_{\text {lon }}$ and $\hat{B}_{\text {lat }}$ [i.e., see (13) and (12)] were selected with values that vary by as much as $40 \%$ from nominal, and (17) is still satisfied (for details on $\hat{B}_{\text {lon }}$ and $\hat{B}_{\text {lat }}$, see [9]).

Simulation parameters were selected based on detailed data analyses and specifications. The sensor noise values are based upon Cloud Cap Technology's Piccolo Autopilot and analysis of data logged during straight and level flight. These values are also corroborated with the specifications given for Cloud Cap Technology's Crista Inertial Measurement Unit (IMU). The thrust limit and estimated rate limit were measured via a static test 

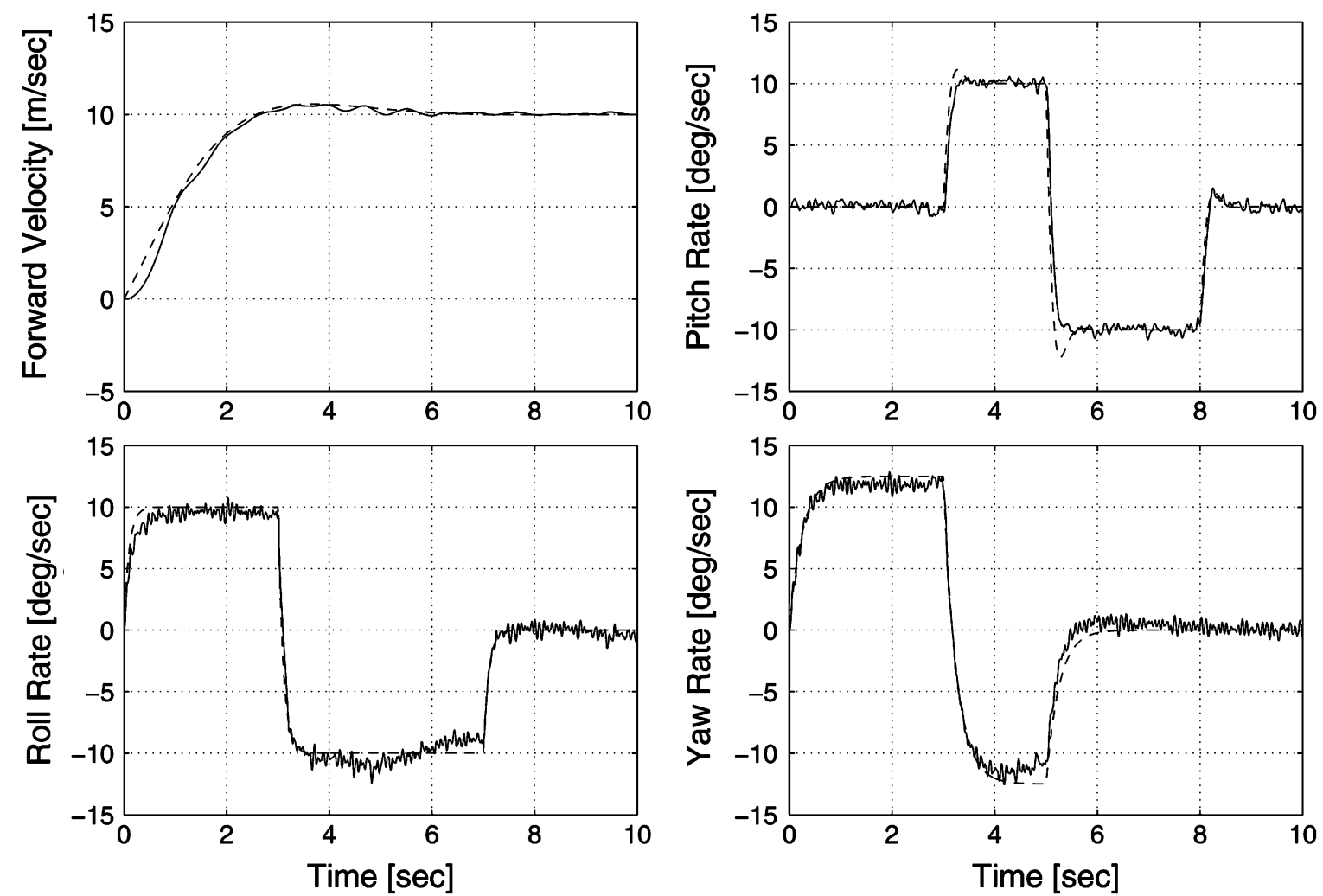

Fig. 2. Model reference (dashed line) and actual (solid line) forward velocity (top left), pitch rate (top right), roll rate (bottom left), and yaw rate (bottom right) responses for closed-loop robust longitudinal and lateral controllers.
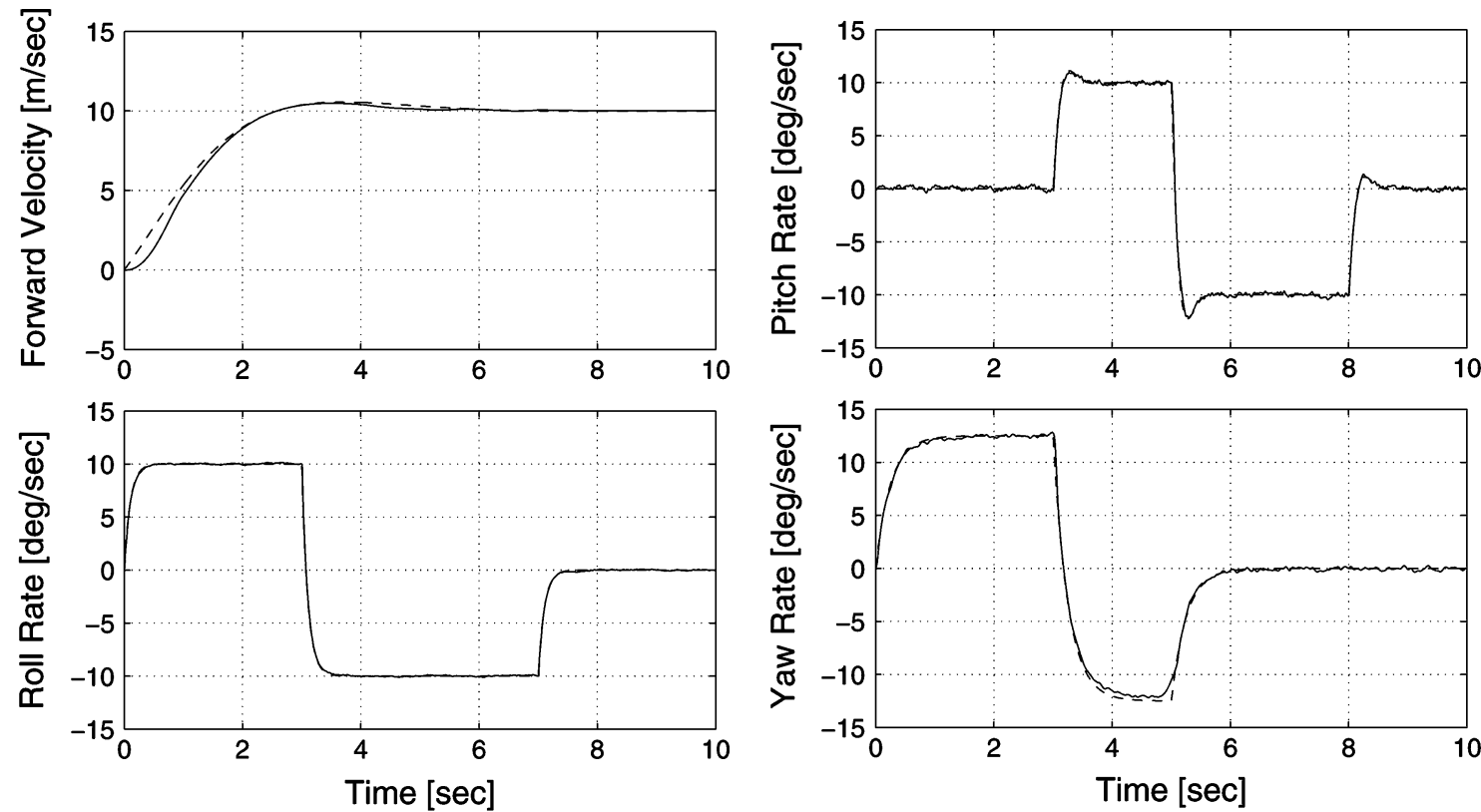

Fig. 3. Model reference (dashed line) and actual (solid line) forward velocity (top left), pitch rate (top right), roll rate (bottom left), and yaw rate (bottom right) responses for closed-loop adaptive longitudinal and lateral controllers.

using a fish scale. The control surface rate and position limits were determined via the geometry of the control surface linkages in conjunction with the detailed specifications sheet given with the Futaba S3010 standard ball bearing servo. For details on the actual simulation parameters, see [9] and [43].

The objectives for the longitudinal controller simulation are to track pitch rate and forward velocity commands. For the lateral controller simulation, the objectives are to track roll rate and yaw rate commands. The reference commands used in the robust and adaptive controller simulations are shown in Fig. 1. The control gains for the robust and adaptive longitudinal and lateral controller simulations [e.g., see (13), and (42)-(44)] were selected such that the controllers exhibit the best possible performance. Fig. 2 shows the forward velocity, pitch rate, roll rate, and yaw rate responses for the closed-loop robust longitudinal and lateral controller simulations. Fig. 3 shows the for- 
ward velocity, pitch rate, roll rate, and yaw rate responses of the closed-loop adaptive systems.

\section{B. Discussion of Results}

Based on the results from the pitch rate and forward velocity simulation, the adaptive controller is capable of achieving better tracking of the maneuvers tested in the simulation. Specifically, the closed-loop system with the adaptive controller exhibits less chattering and greater tracking accuracy than that of the robust controller. The excessive chattering exhibited by the robust controller is likely due to the additional signum term in the corresponding control law. The rms error calculated for the adaptive lateral controller simulation are significantly lower than those calculated for the robust lateral controller. The results show that the rms values of the tracking errors can be reduced significantly (i.e., by more than $94 \%$ ) with a marginal increase in the control effort (i.e., less than 5\%).

\section{CONCLUSION}

A robust aircraft controller is presented, which achieves asymptotic tracking control of a model reference system where the plant dynamics contain input uncertainty and a bounded non-LP disturbance. The developed controller exhibits the desirable characteristic of tracking the specified decoupled reference model. An example of such a decoupling is demonstrated by examining the aircraft response to tracking a roll rate command while simultaneously tracking a completely unrelated yaw rate command. This result represents application of the RISE control strategy in a DI and MRAC framework to a nonlinear system with additive, non-LP disturbances, where the control input is multiplied by a non-square matrix containing parametric uncertainty. To achieve the result, a novel robust control technique is combined with a RISE control structure. An adaptive control extension is also presented, which achieves an asymptotic output tracking result. The extension represents the application of a continuous control strategy in an ADI framework to a nonlinear system with additive, non-LP disturbances, where the control input is multiplied by a non-square matrix containing parametric uncertainty. A Lyapunov-based stability analysis is provided to verify the theoretical result, and numerical simulation results are provided, which demonstrate the robustness of the controllers to sensor noise, exogenous perturbations, parametric uncertainty, and plant nonlinearities, while simultaneously exhibiting the capability to emulate a reference model designed offline. Future work will focus on extending this control design to handle systems of arbitrary relative degree, perhaps by building on the result in [21], [29]. In addition, future efforts will focus on control designs for the considered class of problems where there are fewer inputs than outputs (i.e., where $u(t) \in R^{p}$ and $C \in R^{m \times n}$ with $p<m$ ).

\section{REFERENCES}

[1] M. W. Oppenheimer and D. B. Doman, "Control of an unstable, nonminimum phase hypersonic vehicle model," in Proc. IEEE Aerosp. Conf., Big Sky, MT, Mar. 2006, pp. 1-7.
[2] S. Onori, P. Dorato, S. Galeani, and C. Abdallah, "Finite time stability design via feedback linearization," in Proc. Conf. Decision Control, Eur. Control Conf., Seville, Spain, Dec. 2005, pp. 4915-4920.

[3] Z. Szabo, P. Gaspar, and J. Bokor, "Tracking design for Wiener systems based on dynamic inversion," in Proc. of Int. Conf. Control Appl., Munich, Germany, Oct. 2006, pp. 1386-1391.

[4] J. Chen, D. Li, X. Jiang, and X. Sun, "Adaptive feedback linearization control of a flexible spacecraft," in Proc. Conf. Intell. Syst. Design Appl., Jinan, China, Oct. 2006, pp. 225-230.

[5] A. D. Ngo and D. B. Doman, "Dynamic inversion-based adaptive/reconfigurable control of the X-33 on ascent," in Proc. IEEE Aerosp. Conf., Big Sky, MT, Mar. 2006, pp. 2683-2697.

[6] M. D. Tandale and J. Valasek, "Adaptive dynamic inversion control of a linear scalar plant with constrained control inputs," in Proc. Amer. Control Conf., Portland, OR, Jun. 2005, pp. 2064-2069.

[7] N. Hovakimyan, E. Lavretsky, and C. Cao, "Dynamic inversion of multi-input nonaffine systems via time-scale separation," in Proc. Amer. Control Conf., Minneapolis, MN, Jun. 2006, pp. 3594-3599.

[8] J. Buffington and A. Sparks, "Comparison of dynamic inversion and LPV tailless flight control law designs," in Proc. Amer. Control Conf., Philadelphia, PA, Jun. 1998, vol. 2, pp. 1145-1149.

[9] W. MacKunis, M. K. Kaiser, P. M. Patre, and W. E. Dixon, "Asymptotic tracking for aircraft via an uncertain dynamic inversion method," in Proc. Amer. Control Conf., Seattle, WA, Jun. 2008, pp. 3482-3487.

[10] Q. Wang and R. F. Stengel, "Robust nonlinear flight control of a highperformance aircraft," IEEE Trans. Control Syst. Technol., vol. 13, no. 1, pp. 15-26, Jan. 2005.

[11] T. Yamasaki, H. Sakaida, K. Enomoto, H. Takano, and Y. Baba, "Robust trajectory-tracking method for UAV guidance using proportional navigation," in Proc. Int. Conf. Control, Autom. Syst., Seoul, Korea, Oct. 2007, pp. 1404-1409.

[12] Z. Liu, F. Zhou, and J. Zhou, "Flight control of unpowered flying vehicle based on robust dynamic inversion," in Proc. Chinese Control Conf., Heilongjiang, China, Aug. 2006, pp. 693-698.

[13] X.-. J. Liu, F. Lara-Rosano, and C. W. Chan, "Model-reference adaptive control based on neurofuzzy networks," IEEE Trans. Syst., Man, Cybern. C, Appl. Rev., vol. 34, no. 3, pp. 302-309, Aug. 2004.

[14] A. Calise and R. Rysdyk, "Nonlinear adaptive flight control using neural networks," IEEE Control Syst. Mag., vol. 18, no. 6, pp. 14-25, Dec. 1998.

[15] J. Leitner, A. Calise, and J. V. R. Prasad, "Analysis of adaptive neural networks for helicopter flight controls," J. Guid., Control Dyn., vol. 20, no. 5, pp. 972-979, Sep. 1997.

[16] Y. Shin, "Neural network based adaptive control for nonlinear dynamic regimes," Ph.D. dissertation, Sch. Mechan. Eng., Georgia Tech. Inst., Atlanta, Nov. 2005.

[17] E. Lavretsky and N. Hovakimyan, "Adaptive compensation of control dependent modeling uncertainties using time-scale separation," in Proc. Conf. Decision Control, Eur. Control Conf., Seville, Spain, Dec. 2005, pp. 2230-2235.

[18] E. N. Johnson and A. J. Calise, "Pseudo-control hedging: A new method for adaptive control," in Workshop Adv. Guid. Control Technol., Redstone Arsenal, AL, Nov. 2000.

[19] B. Xian, D. M. Dawson, M. S. de Queiroz, and J. Chen, "A continuous asymptotic tracking control strategy for uncertain nonlinear systems," IEEE Trans. Autom. Control, vol. 49, no. 7, pp. 1206-1211, Jul. 2004

[20] P. M. Patre, W. MacKunis, C. Makkar, and W. E. Dixon, "Asymptotic tracking for systems with structured and unstructured uncertainties," in Proc. Conf. Decision Control, San Diego, CA, Dec. 2006, pp. 441-446.

[21] Z. Cai, M. S. de Queiroz, and D. M. Dawson, "Robust adaptive asymptotic tracking of nonlinear systems with additive disturbance," IEEE Trans. Autom. Control, vol. 51, no. 3, pp. 524-529, Mar. 2006.

[22] B. Xian, M. S. de Queiroz, and D. M. Dawson, A Continuous Control Mechanism for Uncertain Nonlinear Systems in Optimal Control, Stabilization, and Nonsmooth Analysis. Heidelberg, Germany: SpringerVerlag, 2004.

[23] X. Zhang, A. Behal, D. M. Dawson, and B. Xian, "Output feedback control for a class of uncertain MIMO nonlinear systems with nonsymmetric input gain matrix," in Proc. Conf. Decision Control, Eur. Control Conf., Seville, Spain, Dec. 2005, pp. 7762-7767.

[24] P. M. Patre, W. MacKunis, C. Makkar, and W. E. Dixon, "Asymptotic tracking for systems with structured and unstructured uncertainties," IEEE Trans. Control Syst. Technol., vol. 16, no. 2, pp. 373-379, Mar. 2008.

[25] M. L. McIntyre, W. E. Dixon, D. M. Dawson, and I. D. Walker, "Fault identification for robot manipulators," IEEE Trans. Robot. Autom., vol. 21, no. 5, pp. 1028-1034, Oct. 2005. 
[26] S. Gupta, D. Aiken, G. Hu, and W. E. Dixon, "Lyapunov-based range and motion identification for a nonaffine perspective dynamic system," in Proc. Amer. Control Conf., Minneapolis, MN, Jun. 2006, pp. 4471-4476.

[27] W. E. Dixon, Y. Fang, D. M. Dawson, and T. J. Flynn, "Range identification for perspective vision systems," IEEE Trans. Autom. Control, vol. 48 , no. 12 , pp. $2232-2238$, Dec. 2003

[28] A. Behal, D. M. Dawson, W. E. Dixon, and Y. Fang, "Tracking and regulation control of an underactuated surface vessel with nonintegrable dynamics," IEEE Trans. Autom. Control, vol. 47, no. 3, pp. 495-500, Mar. 2002.

[29] J. Chen, A. Behal, and D. M. Dawson, "Robust feedback control for a class of uncertain MIMO nonlinear systems," IEEE Trans. Autom. Control, vol. 53, no. 2, pp. 591-596, Feb. 2008.

[30] L. Duan, W. Lu, F. Mora-Camino, and T. Miquel, "Flight-path tracking control of a transportation aircraft: Comparison of two nonlinear design approaches," in Proc. Digit. Avionics Syst. Conf., Portland, OR, Oct. 2006, pp. 1-9.

[31] I. Szaszi, B. Kulcsar, G. J. Balas, and J. Bokor, "Design of FDI filter for an aircraft control system," in Proc. Amer. Control Conf., Anchorage, AK, May 2002, pp. 4232-4237.

[32] Department of Transportation, Federal Aviation Regulations-Part 25, Washington, DC, "Airworthiness Standards: Transport category airplanes," 1996.

[33] F. L. Lewis, C. T. Abdallah, and D. M. Dawson, Control of Robot Manipulators. New York, NY: MacMillan, 1993.

[34] B. S. Davis, T. Denison, and J. Kaung, "A monolithic high-g SOIMEMS accelerometer for measuring projectile launch and flight accelerations," in Proc. Conf. Sensors, Vienna, Austria, Oct. 2004, pp. 296-299.
[35] V. Janardhan, D. Schmitz, and S. N. Balakrishnan, "Development and implementation of new nonlinear control concepts for a UA," in Proc. Digit. Avionics Syst. Conf., Salt Lake City, UT, Oct. 2004, p. 12.E.5121-10.

[36] T. Wagner and J. Valasek, "Digital autoland control laws using quantitative feedback theory and direct digital design," J. Guid., Control Dyn., vol. 30, no. 5, pp. 1399-1413, Sep. 2007.

[37] M. Bodson and J. E. Groszkiewicz, "Multivariable adaptive algorithms for reconfigurable flight control," in Proc. Conf. Dec. Control, Lake Buena Vista, FL, Dec. 1994, pp. 3330-3335.

[38] B. J. Bacon, A. J. Ostroff, and S. M. Joshi, "Reconfigurable NDI controller using inertial sensor failure detection \& isolation," IEEE Trans. Aerosp. Electron. Syst., vol. 37, no. 4, pp. 1373-1383, Oct. 2001.

[39] G. Tao, Adaptive Control Design and Analysis, S. Haykin, Ed. New York: Wiley-Interscience, 2003.

[40] D. Dawson, M. Bridges, and Z. Qu, Nonlinear Control of Robotic Systems for Environmental Waste and Restoration. Englewood Cliffs, NJ: Prentice-Hall PTR, 1995.

[41] H. K. Khalil, Nonlinear Systems, 3rd ed. Upper Saddle River, NJ: Prentice-Hall, 2002.

[42] D. Enns and T. Keviczky, "Dynamic inversion based flight control for autonomous RMAX helicopter," in Proc. Amer. Control Conf., Minneapolis, MN, Jun. 2006, pp. 3916-3923.

[43] W. MacKunis, M. K. Kaiser, P. M. Patre, and W. E. Dixon, "Adaptive dynamic inversion for asymptotic tracking of an aircraft reference model," presented at the AIAA Guid., Nav., Control Conf., Honolulu, HI, 2008, AIAA-2008-6792.

[44] J. Roskam and C.-T. E. Lan, Airplane Aerodynamics and Performance, C.-T. E. Lan, Ed., 3rd ed. Lawrence, KS: DAR Corp., 1997. 